\title{
Observation of Electron Coherence and Fabry- Perot Standing Waves at a Graphene Edge
}

\section{Citation}

Allen, Monica T., Oles Shtanko, Ion C. Fulga, Joel I-J Wang, Daniyar Nurgaliev, Kenji Watanabe, Takashi Taniguchi, Anton R. Akhmerov, Pablo Jarillo-Herrero, Leonid S. Levitov, and Amir Yacoby. 2017. Observation of Electron Coherence and Fabry-Perot Standing Waves at a Graphene Edge. Nano Letters 17, no. 12: 7380-7386.

\section{Permanent link}

http://nrs.harvard.edu/urn-3:HUL.InstRepos:41275744

\section{Terms of Use}

This article was downloaded from Harvard University's DASH repository, WARNING: This file should NOT have been available for downloading from Harvard University's DASH repository.

\section{Share Your Story}

The Harvard community has made this article openly available.

Please share how this access benefits you. Submit a story.

Accessibility 


\title{
Observation of Electron Coherence and Fabry-Perot Standing Waves at a Graphene Edge
}

\author{
Monica T. Allen, ${ }^{* \dagger}{ }^{\dagger}$ Oles Shtanko, ${ }^{\ddagger}$ Ion C. Fulga, ${ }^{\S}, \|$ Joel I.-J. Wang, ${ }^{\dagger}$ Daniyar Nurgaliev, ${ }^{\dagger}$ Kenji Watanabe, ${ }^{\perp}$ \\ Takashi Taniguchi, ${ }^{\perp}$ Anton R. Akhmerov, ${ }^{\#}$ Pablo Jarillo-Herrero, ${ }^{\ddagger}$ Leonid S. Levitov, \\ and Amir Yacoby ${ }^{*},, \nabla$
}

${ }^{\dagger}$ Department of Physics, Harvard University, 17 Oxford Street, Cambridge, Massachusetts 02138, United States

${ }^{\ddagger}$ Department of Physics, Massachusetts Institute of Technology, 77 Massachusetts Avenue, Cambridge, Massachusetts 02139, United States

${ }^{\S}$ Department of Condensed Matter Physics, Weizmann Institute of Science, 234 Herzl Street, Rehovot 7610001, Israel

"Institute for Theoretical Solid State Physics, IFW Dresden, 01171 Dresden, Germany

${ }^{\perp}$ Environment and Energy Materials Division, National Institute for Materials Science, 1-1 Namiki, Tsukuba, Ibaraki 305-0044, Japan

\#Kavli Institute of Nanoscience, Delft University of Technology, Lorentzweg 1, 2628 CJ Delft, The Netherlands

${ }^{\nabla}$ Harvard John A. Paulson School of Engineering and Applied Sciences, Pierce Hall, 29 Oxford Street, Cambridge, Massachusetts 02138, United States

Supporting Information

ABSTRACT: Electron surface states in solids are typically confined to the outermost atomic layers and, due to surface disorder, have negligible impact on electronic transport. Here, we demonstrate a very different behavior for surface states in graphene. We probe the wavelike character of these states by Fabry-Perot (FP) interferometry and find that, in contrast to theoretical predictions, these states can propagate ballistically over micron-scale distances. This is achieved by embedding a graphene resonator formed by gate-defined $\mathrm{p}-\mathrm{n}$ junctions within a graphene superconductor-normal-superconductor structure. By combining superconducting Aharanov-Bohm interferometry with Fourier methods, we visualize spatially resolved current flow and image FP resonances due to $\mathrm{p}-\mathrm{n}-\mathrm{p}$ cavity modes. The coherence of the standing-wave edge states is revealed by observing a new family of FP resonances, which coexist with the bulk resonances. The edge resonances have periodicity distinct from that of the bulk states manifest in a repeated spatial redistribution of current on and off the FP resonances. This behavior is accompanied by a modulation of the multiple Andreev reflection amplitude onand-off resonance, indicating that electrons propagate ballistically in a fully coherent fashion. These results, which were not anticipated by theory, provide a practical route to developing electron analog of optical FP resonators at the graphene edge.

KEYWORDS: Ballistic transport, Josephson interferometry, graphene edge states, Fabry-Perot interference, electron optics

Curface states in electronic solids, which occur at the $\checkmark$ material boundaries, have a wide range of interesting properties. The Tamm-Schockley states, which originate from crystal structure termination or band bending, are localized in the top atomic layer and, being hindered by disorder at the surface, do not contribute current-carrying pathways. ${ }^{1,2}$ In contrast, topologically protected surface states in topological insulators or quantum Hall systems allow electrons to propagate coherently without backscattering. ${ }^{3,4}$ However, the necessity of topological protection for the coherent propagation of surface states remains an open question. This work reveals the existence of phase-coherent electron waves at the graphene edges that propagate ballistically over submicron length scales. We observe these states, and probe their coherence, by studying standing waves formed at the graphene edges embedded in an electronic Fabry-Perot (FP) interferometer.
Electron resonators have so far been explored in the momentum domain, in which Fermi momentum is simply tuned with a gate, but little is known about the spatial distribution of current flow due to the challenge of imaging current paths with submicron resolution. To elucidate the role of edge effects on wave interference in graphene, we measure the interference of standing waves in a Josephson junction and image the real space distribution of supercurrent flow using Fraunhofer interferometry. ${ }^{5}$ By extracting the spatial structure of current-carrying states in the cavity using Fourier methods, our measurements disentangle edge from bulk current flow and highlight the surprising role of the crystal boundaries on electron wave interference.

Received: July 24, 2017

Revised: September 30, 2017

Published: October 18, 2017 
In a coherent electron cavity, electronic transport is governed by quantum interference of electron plane waves rather than diffusive transport. ${ }^{6,7}$ In our system, a pair of $\mathrm{p}-\mathrm{n}$ junctions serve as electronic mirrors, confining electron waves in analogy to the confinement of light within an optical FP cavity. As an added benefit over optical systems, the Fermi wavelength of electrons in the cavity is electrostatically tunable with a gate, moving the quantized energy levels of the cavity in and out of resonance with the Fermi energy of the superconducting leads. The resonances give rise to the wavelength-dependent transmission through the $\mathrm{n}-\mathrm{p}-\mathrm{n}$ cavity, resulting in the critical current oscillations. Specifically, in the absence of magnetic field, a single electron trajectory with an incidence angle $\theta$ and refraction angle $\theta^{\prime}$ within the cavity yields a transmission probability $P=|T(\theta)|^{2}$, where $T(\theta)$ is the $\mathrm{FP}$ transmission amplitude:

$$
T(\theta)=\frac{t_{1}(\theta) t_{2}(\theta) e^{\mathrm{i} D}}{1-r_{1}(\theta) r_{2}(\theta) e^{2 \mathrm{i} D}} \quad D=k L \cos \theta^{\prime}
$$

where $t_{1,2}(\theta)$ and $r_{1,2}(\theta)$ are angle-dependent transmission and reflection amplitudes for the two $\mathrm{p}-\mathrm{n}$ junctions. Here $L$ is the length of the $n-p-n$ cavity, $k$ is the wavenumber, and $D$ is the one-way optical path. The quantities in eq 1 depend on the refraction angle $\theta^{\prime}$, and thus on the gate voltage. The dependence of the amplitudes $t_{1,2}(\theta)$ and $r_{1,2}(\theta)$ on the angle $\theta$ can in principle be obtained from free-electron Hamiltonian describing the $\mathrm{p}-\mathrm{n}$ junction for a given carrier's wavelength.

In the presence of magnetic field $B$, using a Landau gauge with vector potential $\mathbf{A}=(-B y, 0,0)$, the optical path and transmission amplitude are modified by including the Aharonov-Bohm phase:

$$
\begin{gathered}
D^{\prime}=D+\frac{\pi \Phi L}{2 \Phi_{0} W} \tan \theta^{\prime}, T^{\prime}(\theta)=T(\theta) \mathrm{e}^{\mathrm{i} \varphi_{A B}(y)}, \\
\varphi_{\mathrm{AB}}(y)=\pi \frac{\Phi}{\Phi_{0}} \frac{y}{W}
\end{gathered}
$$

where $\varphi_{\mathrm{AB}}$ is the dominant part of the Aharonov-Bohm phase responsible for the Fraunhofer interference. Here $\Phi=B L W$ is the total magnetic flux through the junction, $\Phi_{0}=h c / 2 e$ is the magnetic flux quantum, and $y$ is the position of the trajectory initial point, $0<y<W$. Here, $D$ is an effective optical path derived as a half-sum of one-way optical paths for the rightmoving and left-moving modes in the resonator.

This result can be applied to the superconducting transport through the superconductor-normal-superconductor (SNS) junction mediated by Andreev pairs. For each electron of the pair, the transmission amplitude through the $\mathrm{p}-\mathrm{n}-\mathrm{p}$ junction is described by eq 1 with the $B$-dependent contribution to the phase given in eq 2 . The choice of the model for SNS transport depends on Thouless energy $E_{\mathrm{T}}=\hbar / \tau$, where $\tau \approx L / v_{\mathrm{F}}$ is the traversal time through the junction and $\hbar$ is the Planck's constant. ${ }^{8}$ The SNS junctions studied in our experiment are characterized by $E_{\mathrm{T}}$ values a few times greater than the superconducting gap $\Delta$. It can be shown that in the limit $E_{\mathrm{T}} \gg$ $\Delta$, a single trajectory with electron transmission probability (eq 1) gives a contribution to the supercurrent of the form:

$$
\begin{array}{r}
I(\varphi, \theta)=\frac{2 e \Delta}{\hbar} \frac{|T(\theta)|^{2} \sin \varphi}{\sqrt{1-|T(\theta)|^{2} \sin ^{2}(\varphi / 2)}} \\
\varphi=\varphi_{1}-\varphi_{2}+2 \varphi_{\mathrm{AB}}\left(y+\frac{1}{2} L \tan \theta^{\prime}\right)
\end{array}
$$

where the phase difference $\varphi$ includes the difference of superconducting phases in the leads $\varphi_{1,2}$ and the AharonovBohm phase gained by an Andreev pair for the given trajectory (for the derivation of eq 3, see the Supporting Information).

The resulting interference fringes are dominated by the angles for which both the transmission and reflection coefficients are reasonably high (the first harmonic of FP fringes is at its brightest when the product of transmission and reflection coefficients $|t(\theta)|^{2}|r(\theta)|^{2}$ takes a maximum value). In general, the spread of angles for different trajectories in the graphene bulk gives rise to a spread of the FP oscillation periods, somewhat reducing the fringe visibility in the net current (see the Supporting Information). In contrast, no suppression is expected for the interference fringes due to edge modes, as discussed in detail later.

The experimental manifestation of these standing-wave edge states constitutes a departure from conventional Josephson behavior and arises from the interplay between superconductivity and electron-optics. While the ballistic Josephson effect has been previously probed in one-dimensional systems such as carbon nanotubes and nanowires, the two-dimensional structure of graphene enables the use of superconducting Aharonov-Bohm interferometry to understand the contributions of electronic pathways at the edge and in the bulk. In this work, we demonstrate how the presence of an electron resonator inside the Josephson junction can induce novel superconducting phenomena, including unconventional Josephson interference patterns that exhibit lobes as a function of Fermi energy and modulation of the multiple Andreev reflection amplitude depending on the interference conditions of electron waves in the cavity.

We employ proximity induced superconductivity to shed light on the microscopic nature of electron interference along the crystal boundaries of a graphene resonator. ${ }^{9-12}$ Embedding the resonator between a pair of superconducting electrodes is the key step that allows the extraction of spatial information on current flow. On a practical level, graphene provides an accessible interface for superconducting electrodes because it is purely a surface material, unlike two-dimensional electron gases embedded in semiconductor heterostructures. Although graphene is not intrinsically superconducting, proximityinduced superconductivity can be mediated by a phasecoherent Andreev reflection at the graphene-superconductor interface. $^{13,14}$ This process features an electron-hole conversion by the superconducting pair potential that switches both spin and valley to preserve singlet pairing and zero total momentum of the Cooper pair. ${ }^{15}$ In this study, we employ gated mesoscopic Josephson junctions consisting of bilayer graphene suspended between two superconducting $\mathrm{Ti} / \mathrm{Al}$ electrodes as well as a graphene device on hBN. The superconductors serve three roles: (1) they create electrostatic potentials that confine electron waves, serving as electronic analogs to mirrors; (2) superconducting interferometry can extract spatial information on how current flows through the system; and (3) beyond equilibrium, scattering events between the superconductors and graphene (multiple Andreev reflec- 

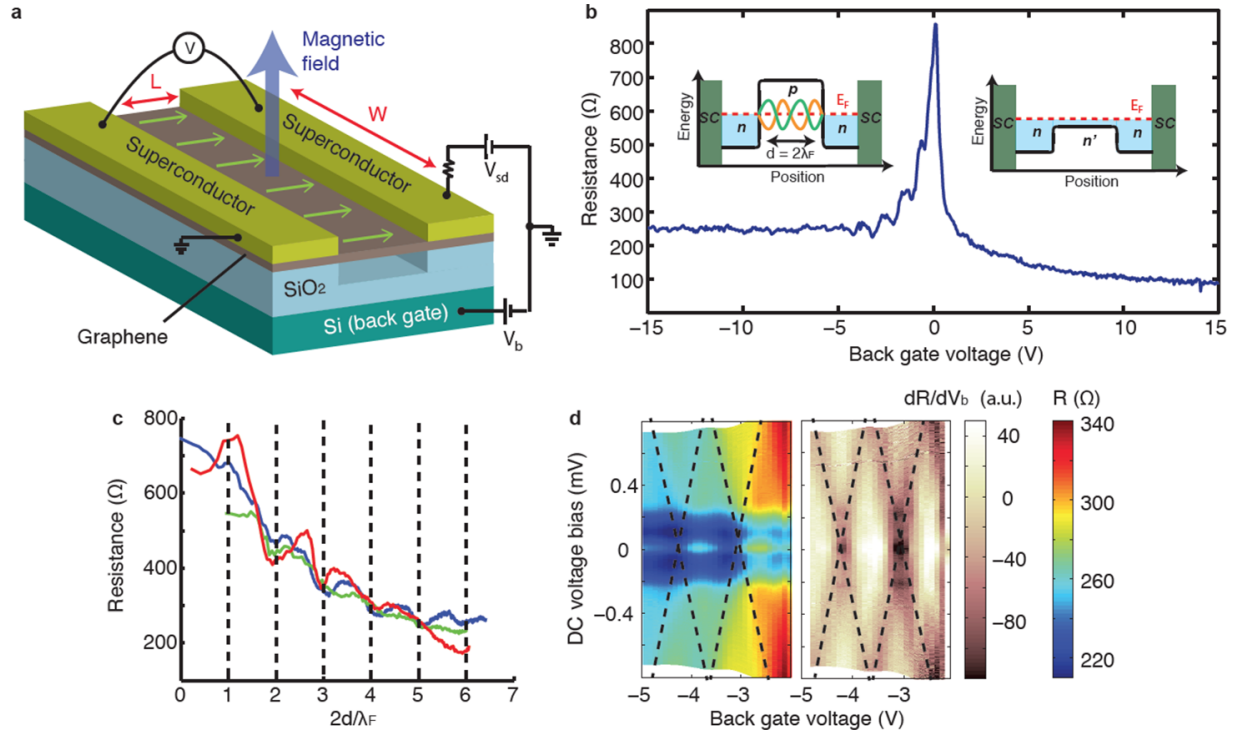

Figure 1. Signatures of the normal-state electron wave interference in a graphene Fabry-Pérot cavity (a) Gated Josephson junction consisting of bilayer graphene suspended between two superconducting electrodes. $L$ is the distance between contacts, and $W$ is the junction width. In the presence of magnetic field, a flux threads the junction area. A voltage applied to the back gate electrode $V_{\mathrm{b}}$ tunes the Fermi wavelength $\lambda_{\mathrm{F}}$ in the cavity. (b) Normal resistance $R_{\mathrm{n}}$, obtained by sweeping the gate voltage $V_{\mathrm{b}}$ at a fixed bias exceeding $I_{\mathrm{c}}$. Data sets in panels $\mathrm{b}-\mathrm{d}$ are from device B1. Left inset: charge transfer at the boundaries of the superconducting electrodes leads to intrinsic $n$-doped regions near the contacts, forming an electronic resonator when the bulk is tuned to hole doping. Dips in resistance appear when constructive interference conditions in the cavity, $2 L=$ $m \lambda_{\mathrm{F}}$, are satisfied for any integer $m$. Right inset: when the bulk is tuned to electron doping, standing waves are not formed, leading to monotonic resistance. (c) $R_{\mathrm{n}}$ plotted versus $2 d / \lambda_{\mathrm{F}}$, where $d$ is the effective junction length and $\lambda_{\mathrm{F}}$ is the Fermi wavelength. By comparing the junction length $L$ to the effective size $d$ extracted from fits, we determined that the contact-doped regions extend at most $100 \mathrm{~nm}$ into the channel, consistent with the results of scanning photocurrent studies. Reproducibility of the oscillation period is demonstrated in three devices of length $L=500 \mathrm{~nm}$. The blue resistance curve is from sample B1, the green curve is from sample B4, and the red curve is from sample $B 5$ and offset by $-250 \Omega$. Resonances marked by dips in resistance appear when constructive interference conditions are satisfied. (d) Fabry-Pérot diamonds obtained using voltage bias spectroscopy, as shown in color maps of $R(\Omega)$ and its derivative $\mathrm{d} R_{\mathrm{n}} / \mathrm{d} V_{\mathrm{b}}$, as a function of back gate voltage $V_{\mathrm{b}}$ and voltage bias $V_{\mathrm{DC}}$. Data are from sample B1.

tions) depend critically on resonance conditions and reveal how the resonator couples to the outside world.

A schematic of a suspended graphene Josephson junction is provided in Figure 1a. Accessing a regime in which electrons travel ballistically, similar to photons, is indispensable for probing the phase-coherent nature of the edge states. We developed a new method to isolate the flake from charge disorder in the underlying dielectric by suspending it over the back gate electrode, described in the Supporting Information. This approach combines the high purity of suspended devices with superconductivity and enables the creation of ballistic waveguides in which the mean free path $l_{\mathrm{e}}$ of electrons exceeds channel length $L$. Similar results are also obtained on a gatedefined resonator in monolayer graphene encapsulated in hBN, discussed later, which enables a higher degree of electronic control over the cavity while preserving sample quality.

The superconducting leads not only serve as electronic probes but also induce a resonant electron cavity in the scaling limit $l_{\mathrm{e}}>L$ (Figure 1b). ${ }^{6,7}$ The graphene in the immediate vicinity of the $\mathrm{Ti} / \mathrm{Al}$ contact is $\mathrm{n}$-doped by charge transfer, ${ }^{16}$ forming an intrinsic $n-n$ or $n-p$ junction near the interface when the graphene has electron or hole carriers, respectively. Taking advantage of contact-induced doping to define the resonator, the resulting $n-p$ junctions constitute electrostatic barriers that are sharp compared to the electron wavelength and are less complex than gate-defined methods. ${ }^{17-20}$ Analogous to an optical Fabry-Pérot cavity, the $n-p$ junctions serve as the electronic counterparts to mirrors, while the ballistic graphene channel serves as an electron waveguide. The Fermi wavelength of electrons in the cavity, expressed as $\lambda_{\mathrm{F}}=2 \pi / \sqrt{\pi n}$ for a twodimensional Fermi disk with 4-fold degeneracy, is experimentally tunable with a gate voltage $V_{\mathrm{b}}$ that determines the carrier density $n$.

FP resonances in ballistic waveguides arise due to reflection from $\mathrm{p}-\mathrm{n}$ junctions formed near the superconducting leads when the carrier polarity in the graphene region is opposite to the polarity of contact doping. Figure $1 \mathrm{~b}$ shows a plot of the normal resistance $R_{\mathrm{n}}$, obtained by sweeping $V_{\mathrm{b}}$ at a fixed bias exceeding the critical current $I_{c}$. We observe periodic resistance oscillations at small positive carrier densities $\left(V_{\mathrm{b}}<0\right)$ when $\mathrm{n}-$ $\mathrm{p}-\mathrm{n}$ junction formation is favored and monotonic behavior when doping is unipolar. The dips in $R_{\mathrm{n}}$ originate from constructive interference of electron waves in the resonator and therefore coincide with carrier densities satisfying the condition $2 d=m \lambda_{\mathrm{F}}$, where $d$ is the effective cavity length and $m$ is an integer. The correspondence to FP interference conditions can be seen more clearly in Figure $1 \mathrm{c}$, which shows that $R_{\mathrm{n}}$ is periodic as a function of $2 d / \lambda_{\mathrm{F}}$. Reproducibility of the oscillation period is demonstrated in three devices of $500 \mathrm{~nm}$ length (Figure 1c), while shorter junctions exhibit larger periods as expected. Quantum confinement between the cavity "mirrors" gives rise to discrete energy levels with spacing $h v_{\mathrm{F}} /$ $2 d$, where $v_{\mathrm{F}}=\hbar k_{\mathrm{F}} / m^{*}$ is the Fermi velocity and $m^{*}$ is the effective electron mass in bilayer graphene. We evaluate this energy scale to be of the order of $\sim 0.8 \mathrm{meV}$ using the height of FP diamonds, as measured using voltage bias spectroscopy (Figure 1d). 

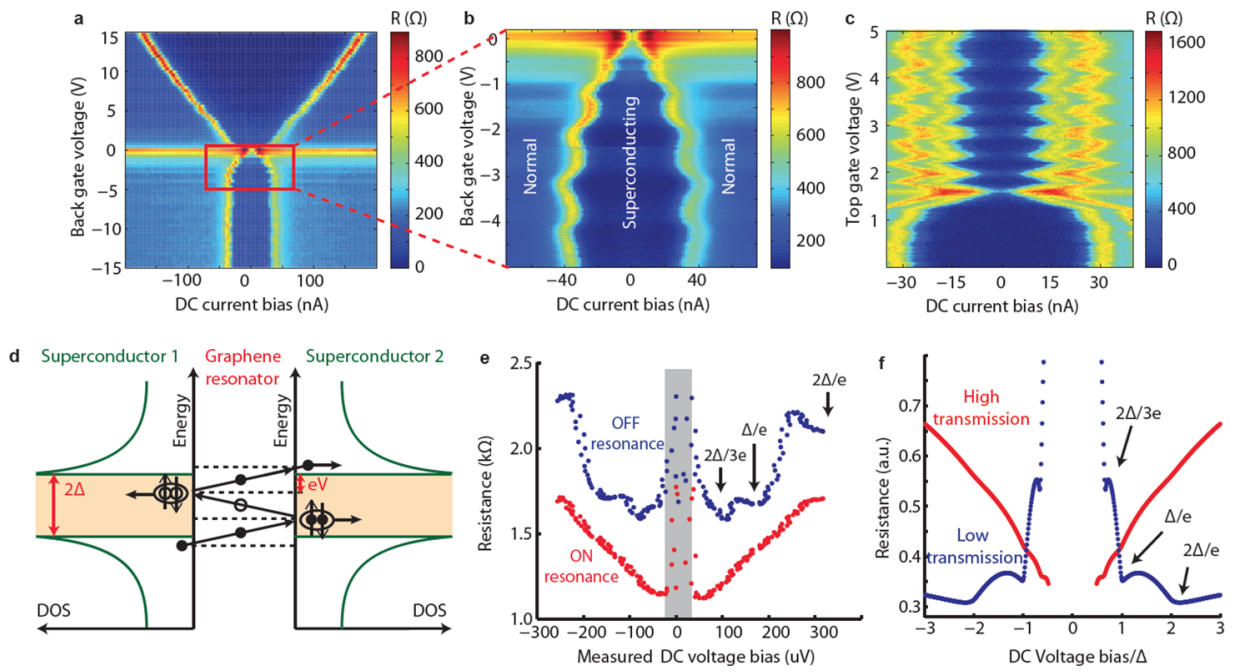

Figure 2. The interplay between superconductivity and the Fabry-Pérot interference in a ballistic graphene Josephson junction. (a,b) Plots of resistance as a function of DC current bias and back gate voltage. The critical current $I_{c}$ oscillates with a period that satisfies the FP interference conditions, consistent with supercurrent propagation via ballistic charge carriers. (c) Differential resistance of a gate-defined FP resonator in monolayer graphene on hBN (device M1) as a function of top gate voltage and DC bias current when the back gate voltage is held fixed at $-1.75 \mathrm{~V}$. The critical current, defined by the width of zero resistance region along the current axis, oscillates with the same periodicity as normal-state resistance, in agreement with a FP model for cavity length of $\sim 100 \mathrm{~nm}$. (d) Schematic illustration of the mechanism of multiple Andreev reflections in a graphene Josephson junction for DC voltage bias $e V=2 \Delta / 3$, which leads to a tilt in the scattering trajectories. (e) Line cuts of resistance versus DC voltage bias "on" ( $V_{\mathrm{b}}=0.3 \mathrm{~V}$, red curve) and "off" $\left(V_{\mathrm{b}}=0.14 \mathrm{~V}\right.$, blue curve) resonance. Well-defined MAR peaks appear at $2 \Delta, \Delta$, and $2 \Delta / 3$ when the density is tuned off resonance, while MAR is suppressed on resonance. Data is from device B3. (f) Modeled conductance profiles in the short junction limit as a function of applied bias voltage. The curve corresponding to high transmission, $G_{\text {high }}$ (red) is computed for a single mode with a transmission of 0.9. The low-transmission curve (blue) is obtained for 4 modes with a transmission of 0.6. Lower transparencies lead to the formation of conductance resonances at bias voltages corresponding to $2 \Delta / 3, \Delta$, and $2 \Delta$.

The interplay between cavity resonances and supercurrent is evident from a resistance colormap as a function of $I_{\mathrm{DC}}$ and $V_{\mathrm{b}}$ (Figure 2a,b) showing critical current oscillations whose period satisfies the FP interference condition, consistent with supercurrent transmitted by ballistic charge carriers. ${ }^{21}$ As $\lambda_{\mathrm{F}}$ in the cavity is tuned with the gate, the quantum levels of the cavity move in and out of resonance with the Fermi energy of the superconducting leads, thus inducing an oscillating critical current periodic in the square root of carrier density. This behavior is observed in two different systems: suspended bilayer graphene resonators defined by contact-induced doping (Figure 2b) and a gate-defined resonator in monolayer graphene on hBN (Figures $2 \mathrm{c}$ and $\mathrm{S} 1$ ), both of which exhibit similar behavior. In total, five suspended bilayer devices are studied with a lithographic distance $L$ between superconducting contacts of 350 to $500 \mathrm{~nm}$ and contact width $W$ of 1.5 to 3.2 $\mu \mathrm{m}$, in addition to one gate-defined monolayer device with cavity dimensions of $L=100 \mathrm{~nm}$ and $W=2.7 \mu \mathrm{m}$ (see the Supporting Information). Figure $2 c$ displays critical current modulations in a gate-defined monolayer resonator whose oscillations are periodic in $n$, in agreement with a monolayer FP for cavity length of $\sim 100 \mathrm{~nm}$.

We employ yet another property of SNS systems to gain insight into the coupling between the cavity modes with the superconducting reservoirs. Because the phenomenon of multiple Andreev reflection (MAR) is known to be extremely sensitive to the coupling between electrons in the normal metal and the superconductor, we use voltage bias spectroscopy to map out the interplay between MAR oscillation amplitude and cavity transmission (Figure 2d,e). The millielectronvolt energy scale associated with FP interference substantially exceeds the $\mathrm{Al}$ superconducting gap $\Delta$, allowing one to study the system close to equilibrium conditions for the resonator. Well-defined
MAR peaks appear at $2 \Delta, \Delta$, and $2 \Delta / 3$ when the density is tuned off resonance, while MAR is completely suppressed on resonance, as visible in line cuts of resistance on and off resonance in Figure 2e (additional data sets are provided in Figures S2-S4). It is notable that the amplitude of the multiple Andreev reflections depends strongly on cavity resonance conditions, thereby providing a direct measure of the tunable coupling between the resonator and the outside world.

We hypothesize that the change in visibility of MAR on and off resonance is most naturally explained by changes in the distribution of transmission eigenvalues. When an electron cavity is tuned to the resonant wavelength, a larger fraction of the current is carried by highly transmitting modes than when the cavity is off resonance. Meanwhile, the magnitude of multiple Andreev reflection peaks should be small for modes with high transmission probability due to the absent suppression of higher order scattering processes. $^{22}$ The interplay between these two effects should thus produce the observed multiple Andreev reflection pattern in short junctions, in which different modes contribute independently to the current. In our devices, the superconducting phase-coherence length $\xi=\hbar v_{\mathrm{F}} / \Delta \approx 450-700 \mathrm{~nm}$, while the channel size is $\sim 350 \mathrm{~nm}$, so we expect the short junction limit to qualitatively hold.

To qualitatively understand the multiple Andreev reflection measurements, we consider a short Josephson junction model. In this limit, the conductance is given by:

$$
G(V)=\int_{0}^{1} \rho(T) G(V, T) \mathrm{d} T
$$

with $T$ the transmission probability, $\rho$ the density of transmission eigenvalues, and $G(T, V)$ the single mode conductance contribution, calculated using expressions from 


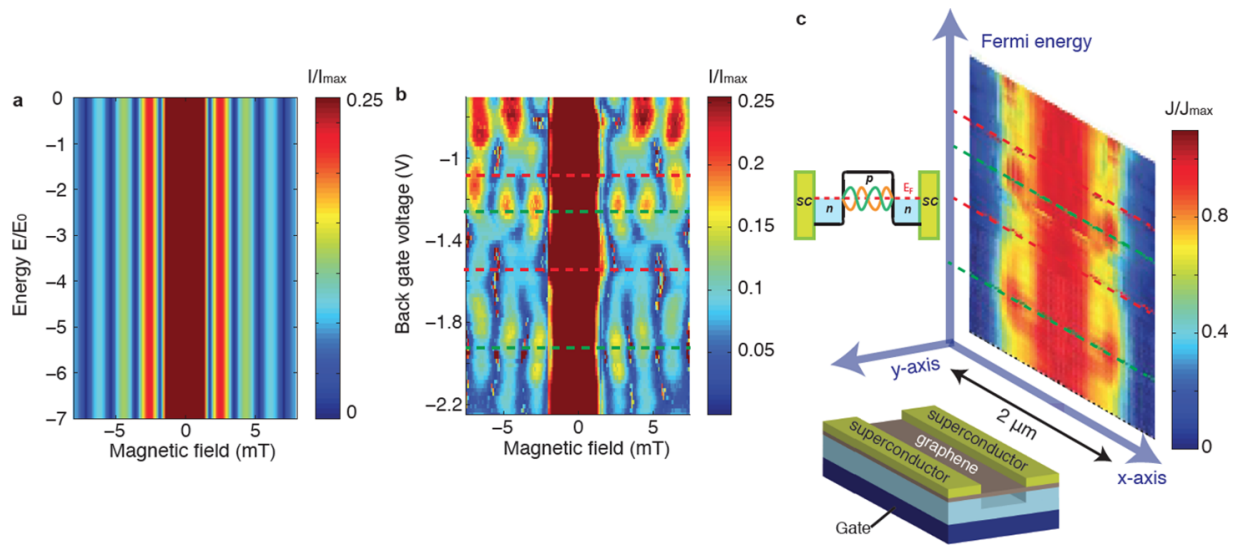

Figure 3. Spatially resolved supercurrent imaging in a ballistic graphene cavity. (a) Theoretical calculation of the superconducting interference pattern for the case of uniform current flow, plotted as the normalized critical current $I_{c}(B) / I_{c}(B=0)$. (b) Plot of normalized critical current $I_{c}(B) / I_{c}$ $(B=0)$, indicating a nontrivial dependence of superconducting interference on cavity resonances. The value of $I_{c}$ at each pixel was obtained by measuring the DC voltage $V_{\mathrm{sd}}$ across the junction as a function as a function of DC current bias $I_{\mathrm{DC}}$ and extracting the maximum derivative $\mathrm{d} V_{\mathrm{sd}} /$ $\mathrm{d} I_{\mathrm{DC}}$. Red and green dotted lines indicate "on" and "off" resonance conditions for the cavity, respectively. Data was collected from device B2. (c) Real-space normalized supercurrent density distribution $J(x) / J_{\max }(x)$ extracted from the $I_{c}(B)$ data in panel b using Fourier techniques $($ see the Supporting Information for details).

ref 22. Because supercurrent is predominately transmitted by bulk modes, as indicated by the Fraunhofer interferometry data (Figure S6), we simplify our MAR analysis by focusing on resonances of bulk states. We approximate the integral as a sum, $G(V) \approx \sum_{n} \alpha_{n} G\left(T_{n}, V\right)$, where:

$$
\alpha_{n}=\int_{T_{n}-T_{n-}}^{T_{n}+T_{n+}} \rho(T) \mathrm{d} T
$$

models the contribution of modes having different ranges of transmission probability.

Following refs 22-24, we compute multiple Andreev reflection curves in the single channel short junction limit, $G_{n}(V)=G\left(T_{n}, V\right)$, for a set of transmission probabilities $T_{n} \in$ $\{0.3,0.4, \ldots, 1.0\}$. A comparison with the measured values, $G_{\mathrm{m}}(V)$, is reduced to fitting for the coefficients $\alpha_{n}$, which minimize the linear system of equations, $G_{\mathrm{m}}(V)-\sum_{n} \alpha_{n}$ $G_{n}(V)$, for each value of the back gate voltage, $V_{\text {gate }}$. The theoretical curves have MAR resonances that are sharper than the experimental ones, so the fitting process is improved by smoothing the single channel results by convolution with a Gaussian function (the width of the Gaussian that we used is $0.03)$. To avoid overfitting, we limit the number of coefficients $\alpha_{n}$ to 3 , corresponding to small $T \in[0,0.4], \alpha_{\text {low }} ;$ medium [0.4, $0.8], \alpha_{\text {med }} ;$ and large $[0.8,1]$ transmission, $\alpha_{\text {high. }}$ The resulting function has three fitting parameters and reads:

$$
\begin{aligned}
& G(V)=\alpha_{\text {low }} G(T=0.3, V)+\alpha_{\text {med }} G(T=0.6, V) \\
& \quad+\alpha_{\text {high }} G(T=0.9, V)
\end{aligned}
$$

Both the amount of smoothing and the value of the superconducting gap to which the bias voltage is rescaled are optimized to produce the smallest fit residuals over the entire range of back gate voltages. We obtain curves that are in good agreement with the measured ones. The coefficients $\alpha_{n}$ corresponding to large transmissions increase, while the ones corresponding to low transmissions decrease whenever the system is on resonance, at values of $V_{\text {gate }}$ at which the normalstate conductance is also peaked (see Figure S5c). However, the estimated normal-state conductance $G_{\mathrm{N}}=g_{0} \sum_{n} T_{n} \alpha_{n}$ is smaller than the measured one for all back gate voltages, which may be due to deviations from the short junction theory or the nonlinear behavior of the $\mathrm{p}-\mathrm{n}$ junctions.

Next, we employ superconducting interferometry as a tool to spatially resolve optics-like phenomena associated with electron waves confined within a ballistic graphene Josephson junction. Unlike experiments in one-dimensional systems, ${ }^{9,10,25}$ one can thread flux through the junction and explore the rich interplay between magnetic interference effects and cavity transmission. Upon the application of a magnetic field $B$, a flux $\Phi$ penetrates the junction area and induces a superconducting phase difference $\Delta \varphi(x)=2 \pi \Phi x / \Phi_{0} W$ parallel to the graphenecontact interface, where $\Phi_{0}=h / 2 e$ is the flux quantum, $h$ is Planck's constant, and $e$ is the elementary charge. When a flux penetrates the junction area, the critical current $I_{c}(B)$ exhibits oscillations in magnetic field given by

$$
I_{c}(B)=\left|\int_{-W / 2}^{W / 2} J(x) \cdot e^{2 \pi \mathrm{i} L B x / \Phi_{0}} \mathrm{~d} x\right|
$$

where $L$ is the distance between superconducting electrodes (Figure 1). ${ }^{5,26}$ This integral expression applies in the wide junction limit, relevant for our system, where $L \ll W$ and the current density is only a function of one coordinate. Because the critical current $I_{c}(B)$ equals the magnitude of the complex Fourier transform of the real-space supercurrent distribution $J(x)$, the shape of the interference pattern is determined directly by the spatial distribution of supercurrent across the sample. ${ }^{5,27}$

To visualize current flow associated with interfering electron waves, we examine the supercurrent modulations in a $B$ field that arise from a Fraunhofer interference. In a conventional Josephson junction with uniform current density, the normalized critical current obeys $I_{c}(B) / I_{c}(0)=$ $\mid \sin \left(\pi \Phi / \Phi_{0}\right) /\left(\pi \Phi / \Phi_{0}\right)$. This "single slit" diffraction pattern would be independent of gate voltage, as illustrated in Figure 3a. In contrast, the fringes in our measured pattern are at variance with this theoretical scenario and exhibit nodes in $I_{\mathrm{c}}(B) / I_{\mathrm{c}}(0)$ as a function of both $V_{\mathrm{b}}$ and $B$. This is illustrated in Figures $3 \mathrm{~b}$ and $\mathrm{S} 6$, which reveal the behavior of the normalized interference pattern $I_{\mathrm{c}} / I_{\mathrm{c}}(B=0)$ at gate voltages corresponding to the on-resonance and off-resonance conditions (labeled by 

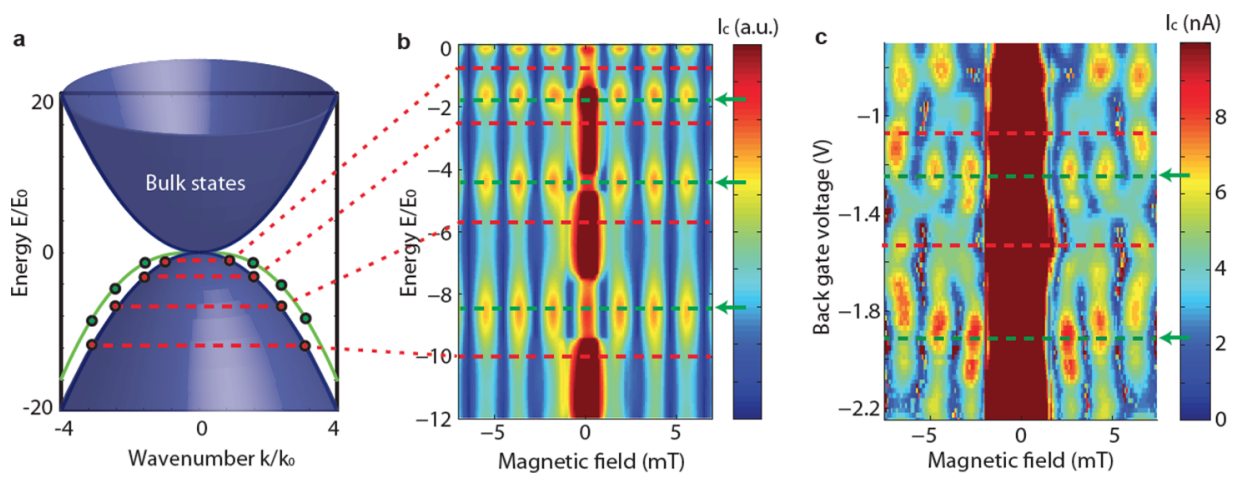

Figure 4. Theoretical model of edge and bulk interference in the ballistic regime. (a) The spectrum of bilayer graphene with a weal edge potential, for which one edge mode dominates. In panels a and $\mathrm{b}, k_{0}=u m^{*} / 2 \hbar$ and $E_{0}=\hbar^{2} k_{0}^{2} / 2 m^{*}$ with $m^{*}=0.028 m_{\mathrm{e}}$ (BLG band mass) and $\Delta$ function potential strength $\hbar u=0.5 \mathrm{eV} \cdot \mathrm{nm}$ (see ref 28). Energies corresponding to quantized momenta are represented by horizontal red lines. (b) Theoretical plot of critical current $I_{c}$ as a function of barrier energy and applied magnetic field in the presence of edge modes. Bulk and edge currents produce distinct FP patterns due to different dispersion laws and the angle-dependent transmission of bulk modes. (c) Critical current $I_{c}$ as a function of back gate voltage $V_{\mathrm{b}}$ and applied magnetic field $B$. Red and green dotted lines indicate "on" and "off" resonance conditions, respectively. Data were collected from device B2.

the red and green dotted lines, respectively). The spatial distribution of supercurrent density $J(x)$, which exhibits gatedependent modulations along both the edge and the bulk of the sample, is provided in Figure S6c. From this figure, we see the on and off modulation of Andreev resonances occurs mostly in the bulk rather than at the edge. We estimate that the supercurrent density per bulk mode exceeds that per edge mode by a factor of 1.5 on when the edge mode is off resonance, becoming approximately equal when the edge mode is on-resonance. This behavior arises because the wave function that composes Andreev states in the bulk have a small overlap with the edge modes.

The reproducibility of this phenomenon in additional samples is demonstrated in Figure S7. To understand the origin of this behavior, we extract an effective spatial distribution of the supercurrent $J(x)$ by taking the inverse Fourier transform of the above $I_{c}(B)$ line plots in accordance with eq 7 and the technique of Dynes and Fulton ${ }^{5}$ (see the Supporting Information). As shown in Figure 3c, the resulting spatial distribution features bulk-dominated current flow on resonance and an enhanced edge current contribution off resonance.

We hypothesize that separate interferences of boundary states and bulk states are responsible for the experimentally observed critical current fringes. Motivated by the relation between the spatial current distribution $J(x)$ and critical current $I_{c}(B)$ in eq 7, we directly model the spatial distribution of current paths for bilayer graphene in the FP regime (see the Supporting Information). These calculations take into account the guided edge modes that originate from edge potentials, which confine carriers to edge-defined "waveguides" in analogy to the confinement of photons in fiber optics. ${ }^{28}$ Energies of the edge modes lie outside the bulk continuum (Figure 4a), which implies an evanescent-wave decay of the modes into the bulk. The resulting modes are quasi one-dimensional, propagating ballistically as plane waves along the graphene edges. Applying the FP quantization condition in the $\mathrm{p}-\mathrm{n}-\mathrm{p}$ region leads to a sequence of FP maxima positioned at $k_{n}=\pi n / L$, where $n$ is an integer and $L$ represents the distance between superconducting contacts. Because the FP resonances for the edge modes originate from head-on transmission and reflection, the corresponding FP fringes are much stronger than the angleaveraged FP fringes for the bulk states (Figure S9).

As shown in the theoretical dispersion in Figure 4a, the interference conditions at the edge and in the bulk should not coincide due to the difference in the carrier dispersion at the edge and in the bulk as well as due to the angle dependence of the FP period for bulk carriers. Hence, a gradual increase of doping will trigger repeated switching between the bulkdominated and edge-dominated regimes, with the current distribution switching from spatially uniform to edge like, accordingly. Qualitatively, this would be manifested in the dependence of measured critical current on applied magnetic field as switching between Fraunhofer and more SQUID-like behavior (Figure $4 \mathrm{~b}, \mathrm{c}$ ). In this paper, the term "edgedominated" refers specifically to Fraunhofer interference patterns that feature an enhancement in the critical currents of the side lobes (outside the $B=0$ central lobe) in comparison to the standard $1 / B$ envelope expected for a uniform supercurrent distribution (see Figure $4 \mathrm{~b}$ ).

To relate this scheme with the transport data, we model FP resonances in accordance with the approach described in ref 28. Assuming that the edge potential is sufficiently short-ranged, we approximate it with a delta function and obtain the density of persistent current along the edge (see the Supporting Information). To translate this prediction into an experimentally observable quantity, we model the superconducting interference pattern $I_{c}(B)$ using the energy dispersion relation of edge modes (Figure 4a). This result, plotted in Figure $4 b$, captures the key features of the raw data in Figure 4c, namely the redistribution of current on and off resonance as well as the suppression of the side lobes' intensity on resonance (Figure S8). Thus, the measurements are consistent with a model that features separate FP interference of guided-wave edge currents in parallel to interference of bulk modes. This further suggests that the quasi-one-dimensional edge currents previously observed have ballistic character. Despite the model's simplified nature, which neglects disorder and finite temperature effects, this scheme captures the essential features of the measurements. While the edge potential featured in this simulation accommodates a single edge channel, we note that the number of guided modes may exceed one for stronger potentials. In this 
case, each mode would contribute independently to the interference pattern.

In summary, we utilize different aspects of proximity-induced superconductivity, particularly Fraunhofer interferometry and Andreev scattering, as new tools to resolve optics-like phenomena associated with electron waves confined within a ballistic graphene Josephson junction. This enables the realspace visualization of cavity modes in a graphene FP resonator, which reveals surprising redistribution of current on and off resonance and provides direct evidence of the ballistic nature of guided edge currents. These results constitute a strong departure from conventional Josephson behavior in graphene and motivate the further exploration of new effects at the intersection of superconductivity and optics-like phenomena.

The ballistic character and coherent nature of edge mode transport in a nontopological material such as graphene is a puzzling behavior that calls for novel theoretical ideas going beyond the usual scenarios for topological protection. New theory must explain why ballistic propagation at exceptionally long length scales occurs despite strong intrinsic disorder present at the edge. One possible scenario, the so-called weak guiding mechanism, has been outlined in this work. Ballistic transport at a strongly disordered graphene edge has been entirely overlooked by previous work, both experimental and theoretical. As such, establishing the presence of a new conduction pathway will dramatically boost our understanding of charge transport mechanisms in atomically thin conductors.

\section{ASSOCIATED CONTENT}

\section{S Supporting Information}

The Supporting Information is available free of charge on the ACS Publications website at DOI: 10.1021/acs.nanolett.7b03156.

Additional experimental details and equations. Figures showing ballistic resistance oscillations, experimental amplitude dependence, resonance interplay, reflection data sets, theoretic reflection amplitude dependence, nontrivial current flow, interference dependence, system configuration, critical current versus the magnetic field, absolute volume of density, and plots of supercurrent density distribution. (PDF)

\section{AUTHOR INFORMATION}

\section{Corresponding Authors}

*E-mail: mtallen@stanford.edu.

*E-mail: yacoby@physics.harvard.edu.

\section{Notes}

The authors declare no competing financial interest.

\section{ACKNOWLEDGMENTS}

The authors thank O. Dial, B. Halperin, V. Manucharyan, and J. Sau for helpful discussions. This work is supported by the Center for Integrated Quantum Materials (CIQM) under NSF award no. 1231319 (L.S.L. and O.S.) and the U.S. DOE Office of Basic Energy Sciences, Division of Materials Sciences and Engineering under award no. DE-SC0001819 (P.J.H., M.T.A., and A.Y.). Nanofabrication was performed at the Harvard Center for Nanoscale Systems (CNS), a member of the National Nanotechnology Infrastructure Network (NNIN) supported by NSF award no. ECS-0335765. A.A. was supported by the Foundation for Fundamental Research on Matter (FOM), The Netherlands Organization for Scientific
Research (NWO/OCW). I.C.F. was supported by the European Research Council under the European Union's Seventh Framework Programme (FP7/2007-2013) and ERC Project MUNATOP, the United States-Israel Binational Science Foundation, and the Minerva Foundation.

\section{REFERENCES}

(1) Tamm, I. Phys. Z. Soviet Union 1932, 1, 733.

(2) Shockley, W. Phys. Rev. 1939, 56, 317.

(3) Büttiker, M. Phys. Rev. B: Condens. Matter Mater. Phys. 1988, 38, 9375-9389.

(4) Hasan, M. Z.; Kane, C. L. Rev. Mod. Phys. 2010, 82, 3045-3067.

(5) Dynes, R. C.; Fulton, T. A. Phys. Rev. B 1971, 3, 3015.

(6) Liang, W.; Bockrath, M.; Bozovic, D.; Hafner, J.; Tinkham, M.; Park, H. Nature 2001, 411, 665-669.

(7) Wu, Y.; Perebeinos, V.; Lin, Y.; Low, T.; Xia, F.; Avouris, P. Nano Lett. 2012, 12, 1417-1423.

(8) Beenakker, C. W. J. Phys. Rev. Lett. 1991, 67, 3836-3839.

(9) Nishio, T.; Kozakai, T.; Amaha, S.; Larsson, M.; Nilsson, H. A.; $\mathrm{Xu}, \mathrm{H}$. Q.; Zhang, G.; Tateno, K.; Takayanagi, H.; Ishibashi, K. Nanotechnology 2011, 22, 445701.

(10) Jorgensen, H. I.; Grove-Rasmussen, K.; Novotny, T.; Flensberg, K.; Lindelof, P. E. Phys. Rev. Lett. 2006, 96, 207003.

(11) Calado, V. E.; Goswami, S.; Nanda, G.; Diez, M.; Akhmerov, A. R.; Watanabe, K.; Taniguchi, T.; Klapwijk, T. M.; Vandersypen, L. M. Nat. Nanotechnol. 2015, 10, 761.

(12) Shalom, M. B.; Zhu, M.; Falko, V. I.; Mishchenko, A.; Kretinin, A.; Novoselov, K.; Woods, C.; Watanabe, K.; Taniguchi, T.; Geim, A.; Prance, J. R. Nat. Phys. 2015, 12, 318-322.

(13) Heersche, H. B.; Jarillo-Herrero, P.; Oostinga, J. B.; Vandersypen, L. M. K.; Morpurgo, A. F. Nature 2007, 446, 56-59.

(14) Du, X.; Skachko, I.; Andrei, E. Y. Phys. Rev. B: Condens. Matter Mater. Phys. 2008, 77, 184507.

(15) Beenakker, C. W. J. Rev. Mod. Phys. 2008, 80, 1337-1354.

(16) Blake, P.; Yang, R.; Morozov, S. V.; Schedin, F.; Ponomarenko, L. A.; Zhukov, A. A.; Nair, R. R.; Grigorieva, I. V.; Novoselov, K. S.; Geim, A. K. Solid State Commun. 2009, 149, 1068-1071.

(17) Young, A. F.; Kim, P. Nat. Phys. 2009, 5, 222-226.

(18) Grushina, A. L.; Ki, D.; Morpurgo, A. Appl. Phys. Lett. 2013, 102, 223102.

(19) Rickhaus, P.; Maurand, R.; Liu, M.; Weiss, M.; Richter, K.; Schonenberger, C. Nat. Commun. 2013, 4, 2342.

(20) Allen, M. T.; Martin, J.; Yacoby, A. Nat. Commun. 2012, 3, 934.

(21) Rittenhouse, G. E.; Graybeal, J. M. Phys. Rev. B: Condens. Matter Mater. Phys. 1994, 49, 1182-1187.

(22) Averin, D.; Bardas, A. Phys. Rev. Lett. 1995, 75, 1831-1834.

(23) Bardas, A.; Averin, D. V. Phys. Rev. B: Condens. Matter Mater. Phys. 1997, 56, R8518-R8521.

(24) Chauvin, M.; vom Stein, P.; Esteve, D.; Urbina, C.; Cuevas, J. C.; Yeyati, A. L. Phys. Rev. Lett. 2007, 99, 067008.

(25) Jarillo-Herrero, P.; van Dam, J. A.; Kouwenhoven, L. P. Nature 2006, 439, 953-956.

(26) Tinkham, M. Introduction to Superconductivity; McGraw-Hill Book Co.: New York, NY, 1975.

(27) Hui, H. Y.; Lobos, A. M.; Sau, J. D.; Das Sarma, S. Phys. Rev. B: Condens. Matter Mater. Phys. 2014, 90, 224517.

(28) Allen, M. T.; Shtanko, O.; Fulga, I. C.; Akhmerov, A.; Watanabe, K.; Taniguchi, T.; Jarillo-Herrero, P.; Levitov, L. S.; Yacoby, A. Nat. Phys. 2015, 12, 128-133. 